\title{
Study of the Soret effect in monosaccharide
}

\author{
solutions \\ Pablo Blanco* and Simone Wiegand* \\ Forschungszentrum Jülich GmbH, IFF - Soft Matter, D-52428 Jülich, Germany \\ E-mail: p.blanco@fz-juelich.de; s.wiegand@fz-juelich.de
}

*To whom correspondence should be addressed 


\begin{abstract}
We investigated the thermal diffusion behavior of aqueous solutions of monosaccharides with the infrared thermal diffusion forced Rayleigh scattering (IR-TDFRS) setup. As monosaccharides we studied the three aldohexoses glucose, galactose, mannose and the two ketohexoses sorbose and fructose. All sugars have the same molecular weight but their structure differs as well as some physical properties such as viscosity, density, thermal expansion coefficient and optical rotation. Additionally, we measured the viscosity and the optical rotation of the monosaccharides solutions in the investigated temperature and concentration range. While there is a clear correlation between the structure and the thermal diffusion behavior for alkanes, the situation is much more complicated for the monosaccharides. Nevertheless, as in the case of the alkanes we find a correlation between the thermal diffusion coefficient with the ratio of the thermal expansion coefficient and the kinematic viscosity. We discuss the physical principles, which connect the thermal diffusion behavior with other thermophysical properties and the structure of the different sugars.
\end{abstract}

January 11,2010

\title{
Introduction
}

The understanding of non-equilibrium properties becomes more and more important in the context of biological processes. ${ }^{1-4}$ One of the still not understood non-equilibrium phenomenon is the thermal diffusion. This process occurs, when an initially homogeneous binary mixture is exposed to a temperature gradient. Due to the temperature gradient a mass flow is initiated. This effect is also known as Ludwig-Soret effect. The achieved concentration change in a certain temperature gradient in binary mixtures is described by the Soret coefficient $S_{\mathrm{T}}=D_{\mathrm{T}} / D$, which can be expressed as the ratio between the thermal diffusion coefficient, $D_{\mathrm{T}}$, and the mass diffusion coefficient, $D$.

In the last years many attempts have been made in order to explain the mechanisms of the thermal diffusion process in binary liquid mixtures. The governing coefficients have been correlated with some physical properties such as density, ${ }^{5}$ molecular weight, ${ }^{6}$ moment of inertia, ${ }^{7}$ viscosity, ${ }^{8}$ 
thermal expansion, ${ }^{8}$ molecular structure ${ }^{9}$ and also chemical interactions. ${ }^{10}$ Different combinations have been used to predict the thermal transport coefficients. During the last decade, the interest of studying the Soret effect has increased covering a wide range of research fields like energy, ${ }^{11}$ biology ${ }^{12-14}$ or oil industry ${ }^{15-17}$ among others.

To the best of our knowledge there is no report in the literature about the thermal diffusion transport properties in monosaccharide-water mixtures. There has only been a study in which the Soret coefficient of the polysaccharide dextran ${ }^{18}$ in water has been investigated. They found that the sign change of Soret coefficient could be influenced by adding urea, which opens the hydrogen bonds in a similar manner as an increase of the temperature. Furthermore, monosaccharides are an interesting system class because they allow a systematic shape variation without changing the molar mass, as it is also the case for alkane isomers. In order to discuss structural changes we show the investigated sugars in the open chain form as Fischer projection in Figure 1, although the ring forms are more probable. In contrast to the alkanes the variation in the structure influences their capability to form hydrogen bonds which is reflected by large differences in their solubilities. ${ }^{19}$ Additionally, they are of fundamental interest in biological systems.

Carbohydrates are the most abundant group of natural products. They are our main source of energy. The building blocks of all carbohydrates are sugars and they can be classified according to how many sugar units are combined in one molecule. Monosaccharides, also called simple sugars, are the most basic chemical unit of carbohydrates. In this paper we study a series of hexoses in water: three aldohexoses (glucose, mannose and galactose), and two ketohexoses (fructose and sorbose).

There are two anomeric configurations of sugars: $\alpha$ and $\beta$-form, according to the stereochemical relationship between the anomeric center (typically the carbon next to the oxygen) and the configuration of the most distant stereogenic center. In water exists an equilibrium of different forms such as furanose and pyranose, which are rings with 5 or 6 carbon atoms, respectively. For instance, $67.5 \%$ of fructose builds the $\beta$-pyranosid, $31.5 \%$ the $\beta$-furanosid form and three other forms exist only with a very low probability. In the case of glucose, the distribution is $38 \%$ of 
$\alpha$-glucopyranose and $62 \%$ of $\beta$-glucopyranose, while the content of glucofuranose is below $1 \%{ }^{20}$

The main objective is to study the effect of the molecular structure on the transport properties, as it has been done in the case of branched alkanes. ${ }^{9}$ The results of mass and thermal diffusion and Soret coefficients are presented as function of temperature, mass concentration of sugar and the composition of the monosaccharides.

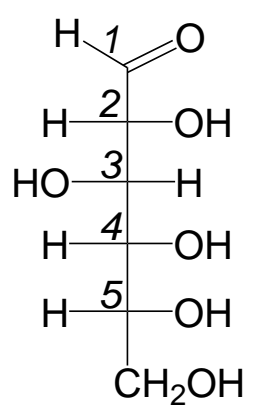<smiles>O=C[C@H](O)[C@H](O)[C@H](O)[C@@H](O)CO</smiles><smiles>O=CC(O)C(O)C(O)C(O)CO</smiles>

\section{D-glucose}

\section{D-mannose}

D-galactose<smiles>O=C(CO)[C@@H](O)[C@H](O)[C@H](O)CO</smiles>

D-fructose<smiles>O=[Z](O)[C@@H](O)[C@H](O)[C@H](O)CO</smiles>

L-sorbose

Figure 1: Fisher projection of the chemical structure of investigated monosaccharides. The numbers identify the different carbon atoms.

\section{Experimental section}

\section{Infrared Thermal Diffusion Forced Rayleigh Scattering}

As experimental method we used the infrared thermal diffusion forced Rayleigh scattering (IRTDFRS) set-up. ${ }^{21}$ The advantage of this setup is that no dye is needed to convert the light energy into heat energy as in the classical TDFRS technique. ${ }^{22-25}$ 
In our IR-TDFRS experiment, the beam of an infrared laser $\left(\lambda_{w}=980 \mathrm{~nm}\right)$ is split into two writing beams of equal intensity which interfere in the sample cell (see Ref. ${ }^{21}$ for a detailed description of the method). Due to a weak absorption band of water around $980 \mathrm{~nm}$ the intensity grating is converted into a temperature grating, which in turn causes a concentration grating by the effect of thermal diffusion. Both gratings contribute to a combined refractive index grating, which is read out by Bragg diffraction of a third laser beam with a wavelength of $\lambda_{r}=633 \mathrm{~nm}$.

A detailed description of the TDFRS technique can be found in the following references. ${ }^{21,26,27}$ The normalized heterodyne intensity $\zeta_{\text {het }}(t)$ is given by

$$
\zeta_{\text {het }}(t)=1+\frac{(\partial n / \partial c)_{p, T}}{(\partial c / \partial T)_{p, c}} S_{\mathrm{T}} c(1-c)\left(1-e^{-q^{2} D t}\right)
$$

where $c$ is the mass concentration, $q$ is the grating vector, whose absolute value is determined by the angle $\theta$ between the two writing beams at the wavelength $\lambda_{w}$ by

$$
q=\frac{4 \pi}{\lambda_{w}} \sin \frac{\theta}{2}
$$

For the determination of the transport coefficients, Eq.(1) is fitted to the measured heterodyne signal using the independently measured contrast factors $(\partial n / \partial c)_{p, T}$ and $(\partial n / \partial T)_{p, c^{*}}$

\section{Materials and Equipment}

D-(+)-glucose (99.5\%), D-(+)-mannose ( $\geq 99 \%)$, D-(+)-galactose $(\geq 99 \%,<0.1 \%$ glucose $)$, D(-)-fructose $(\geq 99 \%,<0.05 \%$ glucose) and L-(-)-sorbose $(\geq 98 \%)$ were purchased from SigmaAldrich. Figure 1 shows the chemical structure of the investigated sugar molecules. We used deionized water from a Millipore Millipak ${ }^{\circledR}$ filter unit $(0.22 \mu \mathrm{m})$.

The mass concentrations of the components have been prepared by weighing with a Mettler Toledo XP504 digital scale with an accuracy of $0.0001 \mathrm{~g}$. Approximately $2 \mathrm{ml}$ of the prepared solutions were filtered through $0.45 \mu \mathrm{m}$ filter (Spartam 13/0.45 RC) before filling them into an optical quartz cell (Hellma) with an optical path length of $0.2 \mathrm{~mm}$. At least three measurements 
Table 1: Thermophysical properties of sugar/water mixtures

\begin{tabular}{|c|c|c|c|c|c|c|c|c|}
\hline sugar & $\begin{array}{c}\text { sugar } \\
\text { weight } \\
\text { fraction } c\end{array}$ & $\begin{array}{c}\text { temper- } \\
\text { ature } \\
T /{ }^{\circ} \mathrm{C}\end{array}$ & $\partial n / \partial c$ & $\begin{array}{c}\partial n / \partial T \\
/ 10^{-4} \mathrm{~K}^{-1}\end{array}$ & $\begin{array}{c}\text { thermal } \\
\text { expansion } \\
\alpha / 10^{-4} \mathrm{~K}^{-1}\end{array}$ & $\begin{array}{c}\text { mixture } \\
\text { density } \\
\rho / \mathrm{gcm}^{-3}\end{array}$ & $\begin{array}{c}\text { kinematic } \\
\text { viscosity } \\
v / \mathrm{cSt}\end{array}$ & $\begin{array}{c}\text { optical } \\
\text { rotation } \\
{[\alpha]_{50} /\left[^{\circ}\right.}\end{array}$ \\
\hline D-fructose & $2 / 3$ & 50 & 0.223 & -2.70 & 6.01 & 1.30267 & 13.264 & \\
\hline D-mannose & $2 / 3$ & 50 & 0.226 & -2.16 & 4.58 & 1.30437 & 14.697 & \\
\hline D-glucose & $2 / 3$ & 50 & 0.224 & -2.13 & 4.52 & 1.30143 & 16.981 & \\
\hline D-glucose & $1 / 2$ & 50 & 0.197 & -2.06 & 4.77 & 1.20844 & 3.370 & \\
\hline D-glucose & $1 / 5$ & 20 & 0.164 & -1.24 & 2.86 & 1.07985 & 1.761 & \\
\hline D-glucose & $1 / 5$ & 30 & 0.163 & -1.49 & 3.58 & 1.07635 & 1.363 & \\
\hline D-glucose & $1 / 5$ & 40 & 0.161 & -1.64 & 4.24 & 1.07217 & 1.091 & \\
\hline D-glucose & $1 / 5$ & 50 & 0.160 & -1.84 & 4.66 & 1.06745 & 0.875 & +22.4 \\
\hline D-fructose & $1 / 5$ & 50 & 0.157 & -1.97 & 5.11 & 1.06793 & 0.846 & -31.8 \\
\hline D-mannose & $1 / 5$ & 50 & 0.162 & -1.86 & 4.71 & 1.06820 & 0.851 & +5.8 \\
\hline D-galactose & $1 / 5$ & 50 & 0.162 & -1.91 & 4.85 & 1.06926 & 0.864 & +30.9 \\
\hline L-sorbose & $1 / 5$ & 50 & 0.155 & -1.91 & 4.91 & 1.06683 & 0.857 & -17.6 \\
\hline
\end{tabular}

with different cells and freshly prepared samples were done for each binary system.

A Tamson TV2000 AKV viscometer has been used to determine the kinematic viscosity of the mixtures. The temperature stability is $\Delta T= \pm 0.01 \mathrm{~K}$. The time can be measured with an accuracy of $\Delta t=0.01 \mathrm{~s}$. Two different Ubbelohde capillaries have been used to measure the viscosities of the mixtures with sugar mass fractions of $c=0.2000$ and $c=0.6667$ at $50{ }^{\circ} \mathrm{C}$. The first capillary has been calibrated with Millipore water at $50{ }^{\circ} \mathrm{C}$, where water has a dynamic viscosity of $\eta=0.5470$ mPas. ${ }^{28}$ The density of water ${ }^{29}$ at $50{ }^{\circ} \mathrm{C}\left(\rho=0.99803 \mathrm{~g} \cdot \mathrm{cm}^{-3}\right)$ is used to convert the kinematic viscosity, $v$, into the dynamic viscosity, $\eta=v \cdot \rho$. The constant of the second capillary was determined with a viscosity standard from Brookfield with a nominal dynamic viscosity of $\eta=4.9 \mathrm{mPas}$ at $25^{\circ} \mathrm{C}$. We assumed that the constant of this second capillary does not change considerably with temperature when it is used at $50{ }^{\circ} \mathrm{C}$. Each viscosity measurement was repeated at least 8 times. The obtained averaged kinematic viscosities are presented in Table 1.

An Anton Paar DMA 4500 densimeter has been used to determine the density, $\rho$, and thermal expansion coefficient, $\alpha$, of the mixtures. The densimeter has an accuracy of $\Delta \rho= \pm 0.00001$ $\mathrm{g} \cdot \mathrm{cm}^{-3}$ and a temperature control of $\Delta T= \pm 0.01 \mathrm{~K}$.

An Anton Paar RXA 156 refractometer has been used to measure the refractive index incre- 
ments with the mass concentration $(\partial n / \partial c)$. The accuracy is of $\Delta n=0.00003$ with a temperature control of $\Delta T= \pm 0.01 \mathrm{~K}$. For each sugar solution we measured the refractive index of five different concentrations around the desired concentration. Linear interpolation of the data provides the slope $(\partial n / \partial c)$. The refractive index increments with temperature $(\partial n / \partial T)$ have been determined with a Michelson interferometer, ${ }^{30}$ in a temperature range of $2 \mathrm{~K}$ above and below the temperature of interest. For all investigated temperatures and concentrations we find a linear dependence of the refractive index versus concentration and temperature, respectively.

The optical rotation of the mixtures has been measured by means of a Jasco P-2000 polarimeter. The temperature stability is of $\Delta T= \pm 0.1 \mathrm{~K}$, and the angular resolution is $\Delta \alpha=0.0001^{\circ}$. The cell is $10 \mathrm{~cm}$ long and its volume is approximately $1 \mathrm{~cm}^{3}$. All solutions were prepared at least 1-2 days before the measurement so that the optical rotation was in equilibrium. The determined values are listed in Table 1, along with the results of the refractive index increments, thermal expansion coefficients, densities, optical rotations and viscosities.

\section{Results and Discussions}

Table 2: Thermal diffusion, mass diffusion and Soret coefficients of sugar/water mixtures

\begin{tabular}{c|c|c|c|c|c}
\hline \hline sugar & $\begin{array}{c}\text { sugar mass } \\
\text { concentration }\end{array}$ & $\begin{array}{c}\text { temperature } \\
/{ }^{\circ} \mathrm{C}\end{array}$ & $\begin{array}{c}S_{\mathrm{T}} \\
/ 10^{-3} \mathrm{~K}^{-1}\end{array}$ & $\begin{array}{c}D \\
/ 10^{-10} \mathrm{~m}^{2} \mathrm{~s}^{-1}\end{array}$ & $\begin{array}{c}D_{T} \\
/ 10^{-13} \mathrm{~m}^{2} \mathrm{~s}^{-1} \mathrm{~K}^{-1}\end{array}$ \\
\hline D-fructose & $2 / 3$ & 50 & $2.47 \pm 0.02$ & $2.60 \pm 0.04$ & $6.43 \pm 0.09$ \\
D-mannose & $2 / 3$ & 50 & $1.54 \pm 0.01$ & $2.65 \pm 0.03$ & $4.08 \pm 0.04$ \\
D-glucose & $2 / 3$ & 50 & $0.84 \pm 0.01$ & $2.57 \pm 0.09$ & $2.16 \pm 0.07$ \\
D-glucose & $1 / 2$ & 50 & $2.26 \pm 0.01$ & $5.08 \pm 0.14$ & $11.5 \pm 0.30$ \\
D-glucose & $1 / 5$ & 20 & $1.10 \pm 0.02$ & $4.30 \pm 0.16$ & $4.73 \pm 0.21$ \\
D-glucose & $1 / 5$ & 30 & $2.08 \pm 0.02$ & $5.69 \pm 0.14$ & $11.8 \pm 0.31$ \\
D-glucose & $1 / 5$ & 40 & $2.71 \pm 0.02$ & $7.36 \pm 0.14$ & $20.0 \pm 0.41$ \\
D-glucose & $1 / 5$ & 50 & $3.26 \pm 0.05$ & $9.24 \pm 0.28$ & $29.9 \pm 0.69$ \\
D-fructose & $1 / 5$ & 50 & $4.99 \pm 0.01$ & $9.60 \pm 0.07$ & $47.9 \pm 0.36$ \\
D-mannose & $1 / 5$ & 50 & $3.77 \pm 0.02$ & $9.26 \pm 0.30$ & $34.9 \pm 1.18$ \\
D-galactose & $1 / 5$ & 50 & $4.18 \pm 0.03$ & $9.10 \pm 0.24$ & $38.1 \pm 1.04$ \\
L-sorbose & $1 / 5$ & 50 & $4.43 \pm 0.01$ & $9.29 \pm 0.20$ & $41.1 \pm 0.94$ \\
\hline \hline
\end{tabular}




\section{Influence of sugar structure and physical properties}

Aqueous solutions of five different monosaccharides have been studied at a sugar weight fraction of $c=0.2$ and at $50{ }^{\circ} \mathrm{C}$. Additionally, three of them have been studied at a sugar weight fraction of $c=0.6667$ and the same temperature. The values of thermal diffusion, mass diffusion and Soret coefficients are listed in Table 2.

The mass diffusion coefficient, $D$, at a weight fraction of $c=0.2$ at $50{ }^{\circ} \mathrm{C}$ is for all five sugars within the error bars identical. The same behavior can be observed for a weight fraction of $c=0.6667$. Therefore, we conclude that the mass diffusion coefficient is independent of the monosaccharide structure, and remains constant for a certain temperature and concentration. As explained in the introduction the different sugars form different tautomers, where the ratio de-

pends on the monosaccharide and varies if the solvent or the temperature is changed. ${ }^{31,32}$ Hence, the thermal properties presented in this study, as a matter of fact, are the average value of different stereoisomers that coexist in equilibrium.

The Soret coefficient is larger for ketohexoses (fructose and sorbose) than for aldohexoses (glucose, mannose and galactose). If the carbonyl group is in the head or tail position of the open chain tautomer, the Soret coefficient is smaller compared to a location within the chain. Due to equal masses of all sugars and also roughly the same hydrodynamic volume, which is reflected by the same diffusion coefficient, we assume that the Soret coefficient changes because of the variation in the molecular structure of the monosaccharides. The position and location of the chiral centers, which are related to the optical rotation, might also be responsible for the thermal diffusive motion of the sugar molecules. Looking at the Fischer projection (c.f. Figure 1), the studied aldohexoses only differ in the hydroxyl groups of the carbon atoms $\mathrm{C}_{2}$ and $\mathrm{C}_{4}$. Apparently, in this simplified open chain cartoon the Soret coefficient is larger when the hydroxyl groups are far apart from the aldehyde group. Contrarily, it is just the opposite in the studied ketohexoses. Fructose and Sorbose only differ in the hydroxyl groups of the carbon atoms $\mathrm{C}_{4}$ and $\mathrm{C}_{5} . S_{\mathrm{T}}$ is larger if the hydroxyl groups are in the same direction as the carbonyl group. Considering the geometry of all tautomer forms makes the discussion even more complicated. 
There is no simple explanation based on the structure of the sugars that could explain the observed differences in the Soret coefficient. In order to gain a better understanding of the Soret coefficient for the different sugars it would be useful to find a correlation with other physical properties, which might be related such as solubility, moment of inertia, ovality and dipole moment. For instance it could be expected that the Soret coefficient becomes higher, when the solubility is lower and the components are less compatible so that a temperature gradient might cause a larger concentration gradient. Also the shape parameter such as the moment of inertia and ovality, which show for other binary organic mixtures a clear correlation ${ }^{7,9,10}$ are not correlated with $S_{\mathrm{T}}$. Also the dipole moment which is a measure for the polarity shows no systematic trend with $S_{\mathrm{T}}$.

Another property which often has been correlated with structural properties is the optical ro$\operatorname{tation}^{33,}{ }^{34}$ Therefore, we measured the optical rotation in order to investigate whether structural changes affect the optical rotation and the thermal diffusion behavior in a correlated way. In order to avoid confusion we want clearly to state that we do not think that the optical rotation and the Soret effect are physically related, but structural changes influence the optical rotation and also the Soret coefficient. Whereas the latter depends not only on the position of the chemical groups but also on their chemical interactions. We measured the optical rotation of the aqueous sugar solutions with weight fraction of $c=0.2$ at $T=50{ }^{\circ} \mathrm{C}$. The results are shown in Table 1 . A direct comparison with literature data is difficult. Several authors ${ }^{35,36}$ measured the optical rotation of various sugars at lower concentrations between 3 and $12 \%$ at ambient temperatures. They observed a similar tendency in the optical rotation (c.f. inset of Figure 2).

In Figure 2 we plotted the Soret coefficient, $S_{\mathrm{T}}$ as a function of the optical rotation at $T=50{ }^{\circ} \mathrm{C}$. Except for galactose the Soret coefficient decreases with increasing value of the optical rotation, $[\alpha]_{50}$. The same trend is observed if one compares the Soret coefficients with the optical rotations determined at ambient temperatures ${ }^{35,36}$ for lower concentrations (c.f. inset of Figure 2). Also here, we observed for galactose the largest positive optical rotation and compared to the trend of the other sugars a too high Soret coefficient. Among the other sugars galactose has the lowest hydrophilicity ${ }^{37}$ and solubility. This low compatibility water might be the reason that a temperature 
gradient causes a larger concentration gradient and results in a larger Soret coefficient compared to the other sugars. This observation indicates that the Soret coefficient of sugars compared to alkanes requires a more sophisticated model covering the positions of the hydration site in relation to the shape of the entire molecules.

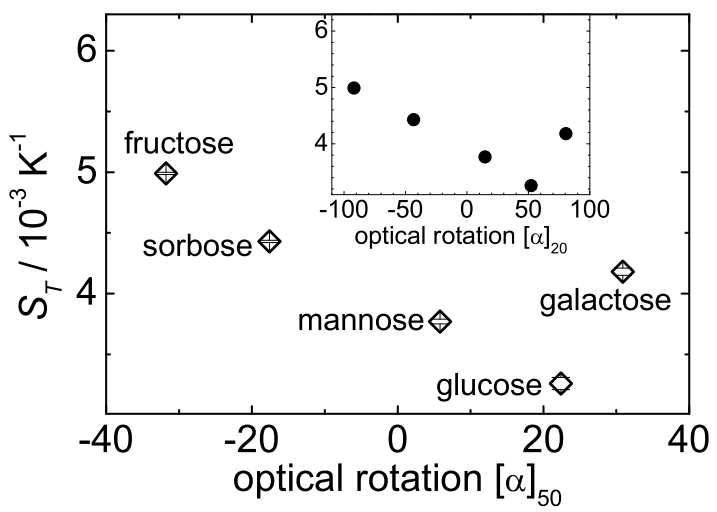

Figure 2: Soret coefficient of sugar in water with $c=0.2$ as function of the optical rotation, $[\alpha]_{50}$, at $50{ }^{\circ} \mathrm{C}$. The inset shows $S_{\mathrm{T}}$ versus literature values of $[\alpha]_{20}$ determined for lower sugar contents between 3 and $12 \%$. $^{35,36}$

The thermal diffusion, the mass diffusion and the Soret coefficient of the monosaccharides as function of the thermal expansion coefficient of the mixtures have been plotted in Figure 3 for the different monosaccharides in water with a weight fraction of $c=0.2$. The thermal diffusion and the Soret coefficient decays linearly with the thermal expansivity. Especially for charged colloidal particles in water, the role of the thermal expansion coefficient of the solvent in relation with the thermal diffusion coefficient has been discussed experimentally and theoretically. ${ }^{38-40}$ Brenner $^{39}$ pointed out that $D_{\mathrm{T}}$ is correlated with the product of solvent diffusion and thermal expansion coefficient. Due to the fact that in our case the diffusion coefficient is constant for all sugars, the thermal expansivity of the solvent seems to be the dominant contribution. Recently, Blanco et al. found for equimolar mixtures of normal alkanes a linear dependence of the thermal diffusion coefficient as function of the ratio between the thermal expansion and viscosity of the mixtures. ${ }^{8}$ Surprisingly, we find a similar tendency for sugar-water mixtures with a weight fraction of $c=0.2$ (c.f. Figure 4 (a)). But at higher concentrations of $c=0.6667$ we find clear deviations from 
a simple linear behavior (c.f. Figure 4 (b)). The importance of the viscosity for the thermal diffusion behavior has been discussed before for diluted polymer solutions. ${ }^{41}$ However, a direct comparison is not possible because we worked with concentrated solutions. The physical reason for a correlation between the thermal expansion coefficient and the thermal diffusion behavior in liquid mixtures might be the following: the thermal expansion coefficient in liquids is dominated by the additional place requirement of the molecules at higher temperatures and the attraction between the molecules. If the latter is weak the thermal diffusion coefficient becomes larger.

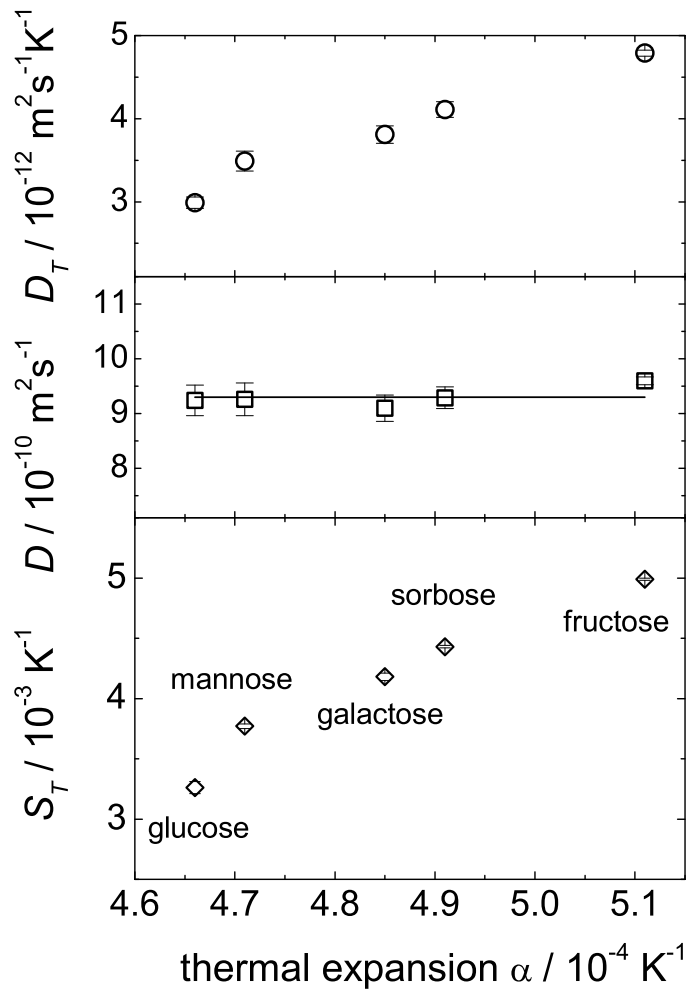

Figure 3: Thermal diffusion, mass diffusion and Soret coefficient of different sugars in water at $50{ }^{\circ} \mathrm{C}$ with a weight fraction $c=0.2$ as function of the thermal expansion coefficient, $\alpha$ of the mixture. The solid line represents the average diffusion coefficient of the five sugars. The error bars represent uncertainty of repeated measurements. 

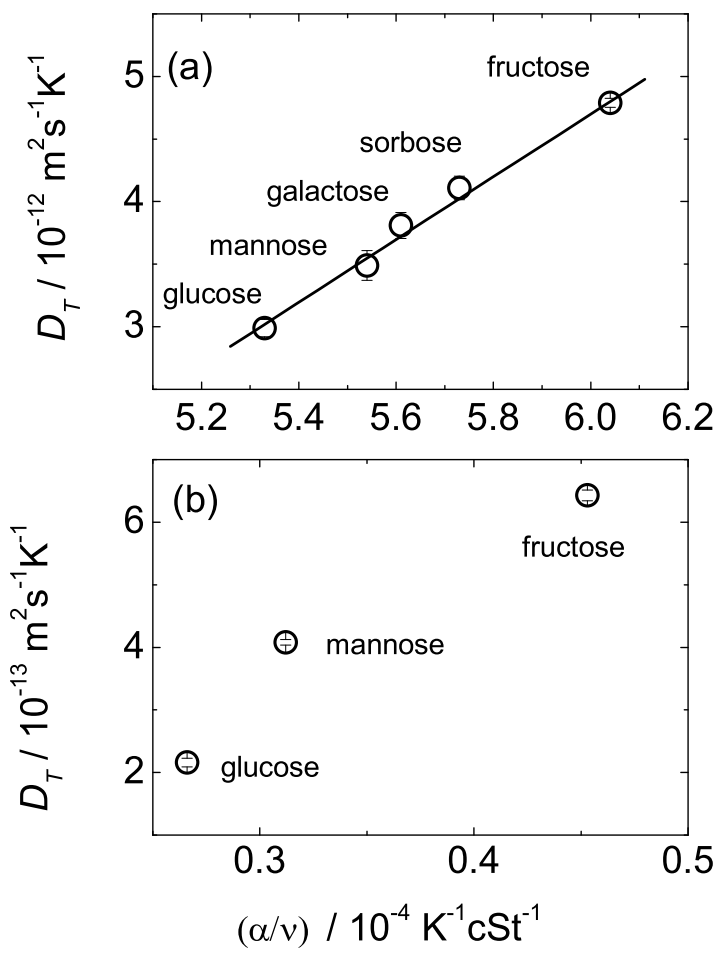

Figure 4: Thermal diffusion of different monosaccharides in water with a weight fraction of $c=0.2$ (a) and $c=0.6667$ (b) at $T=50^{\circ} \mathrm{C}$ as function of the ratio of the thermal expansion coefficient $\alpha$ and the kinematic viscosity $v$. 


\section{Concentration and temperature dependence}

Additionally, we studied the concentration dependence of the thermal diffusion behavior for the system D-glucose/water at a mean temperature of $50{ }^{\circ} \mathrm{C}$. The chosen weight fractions of $c=2 / 3$, $c=1 / 2$ and $c=1 / 5$ correspond to an exact ratio between the number of sugar and water molecules 1 to 5, 1 to 10 and 1 to 40, respectively. Due to the low solubility of D-galactose and L-sorbose in water it was no possible to study those at $c=2 / 3$ and $50{ }^{\circ} \mathrm{C}$. The solubility of galactose is $\approx 47 \%$ wt compared to glucose with $\approx 70 \%$. ${ }^{42}$ The fairly high sugar concentrations, which we used in the experiment should make possible to compare the experimental results with simulations in the future. To achieve a good statistics for the sugar molecules in the simulation it is necessary to go to sufficiently high concentrations. To speed up the dynamic in the simulation it is also useful to work at higher temperatures.

Figure 5 shows the mass diffusion, $D$, thermal diffusion, $D_{T}$, and Soret coefficient, $S_{\mathrm{T}}$, of Dglucose in water at $50{ }^{\circ} \mathrm{C}$ as function of concentration. All three coefficients decay with increasing sugar content. The thermal diffusion coefficient of the high concentrated sugar solutions with $c=$ 0.6667 is one order of magnitude smaller than for the low concentrated solution with $c=0.2$. The observed concentration dependence is similar for all sugar-water systems (c.f. Table 2), although the ratio changes $S_{\mathrm{T}}^{\text {sugar }_{1}} / S_{\mathrm{T}}^{\text {sugar }_{2}}$. For instance the ratio of $S_{\mathrm{T}}$ of fructose and glucose increases from 1.5 to 2.9 by almost a factor of 2 by changing the concentration from $c=0.2$ to $c=0.6667$. The physical reason might be that at higher concentrations the conformation of the sugar molecules is more relevant for the fluid structure so that structural incompabilities result in a larger separation of the sugars in a temperature gradient.

Mogi et al. ${ }^{43}$ measured the mass diffusion coefficients of different diluted aqueous sugar solutions at various temperatures. The extrapolation of their mass diffusion coefficient of D-glucose at $50{ }^{\circ} \mathrm{C}$ is $D=1.23 \cdot 10^{-9} \mathrm{~m}^{2} \mathrm{~s}^{-1}$ while the linear extrapolation of our mass diffusion coefficient of D-glucose to infinite dilution results in $D=1.21 \cdot 10^{-9} \mathrm{~m}^{2} \mathrm{~s}^{-1}$, which is in good agreement with our data. Mogi et al. ${ }^{43}$ observed also a constant mass diffusion coefficient for diluted solutions of glucose, mannose and galactose in water at constant temperature. The maximum deviation from the 
average was $3.8 \%$ in the temperature range from $0{ }^{\circ} \mathrm{C}$ to $80{ }^{\circ} \mathrm{C}$. We come to the same conclusion for glucose, mannose and fructose, if we compare the two sets of different mass concentrations of $c=0.2$ and $c=0.6667$ at $50{ }^{\circ} \mathrm{C}$.

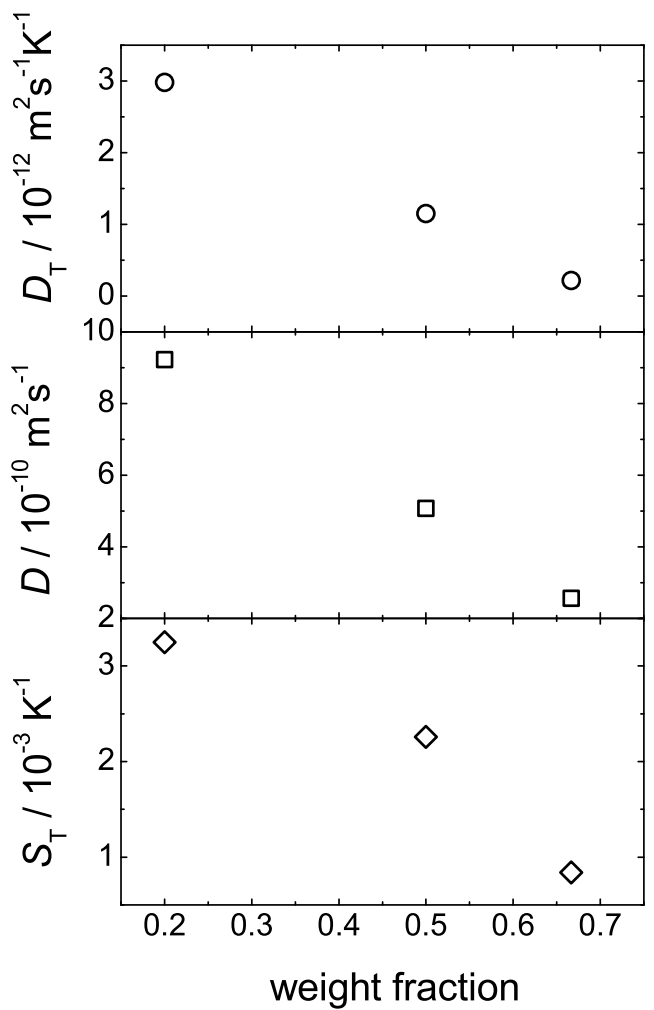

Figure 5: Thermal diffusion, mass diffusion and Soret coefficients of D-glucose/water at $50{ }^{\circ} \mathrm{C}$ as function of the weight fraction.

Figure 6 shows the experimentally determined Soret, mass diffusion and thermal diffusion coefficient as function of temperature for the system D-glucose/water at a sugar weight fraction of $c=0.2$. The mass diffusion coefficient increases in the investigated temperature range roughly by a factor of two, which is mainly caused by the decrease of the viscosity with increasing temperature. The thermal diffusion coefficient $D_{T}$ increases roughly by a factor of six and $S_{\mathrm{T}}$ increases in accordance by a factor of three with temperature. We expect a similar behavior for the other sugars, but due to the fact that the rigidity of the molecules becomes smaller at higher temperatures, the conformational differences are partially overridden so that the thermal diffusion behavior might be 
more similar at high temperatures, but this needs to be studied in further experiments.

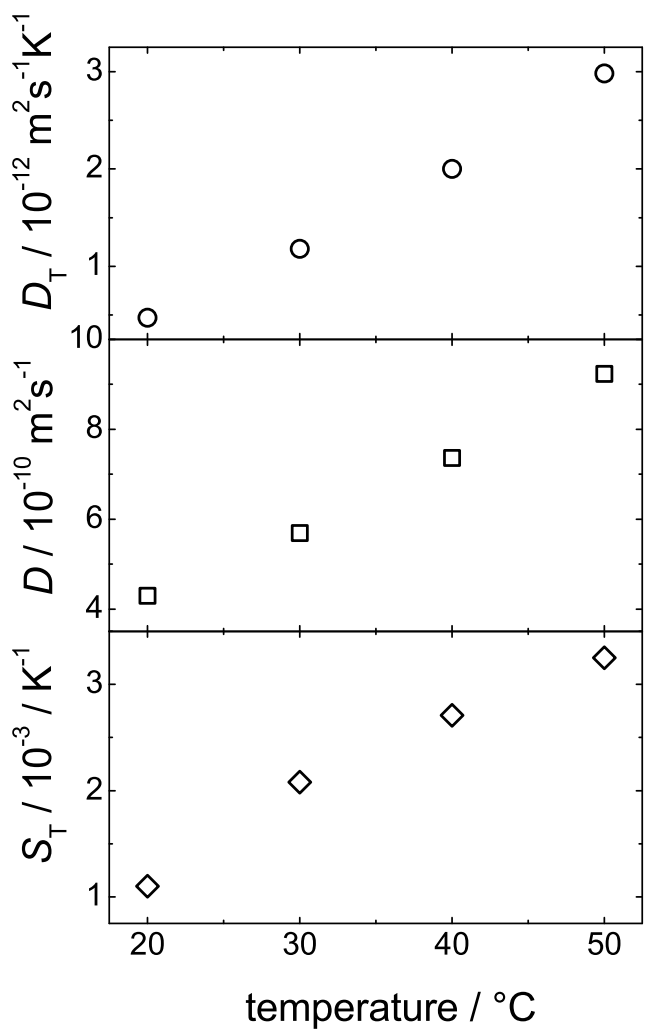

Figure 6: Thermal diffusion, mass diffusion and Soret coefficients of D-glucose/water with a sugar weight fraction $c=0.2$ as function of temperature.

As discussed in the previous section we found for the thermal diffusion coefficient $D_{\mathrm{T}}$ of different sugars in water at the same temperature and concentration a linear dependence as function of the ratio of the thermal expansion coefficient $\alpha$ and the kinematic viscosity $v$ (see Figure 4 ). The same trend we observe for D-glucose in water as shown in Figure 7a comparing the results at different temperatures at a D-glucose weight fraction of $c=0.2$. Regarding the concentration dependence we find deviations from the linear behavior as it was also observed for the different sugars at a higher weight fraction of $c=0.67$. 


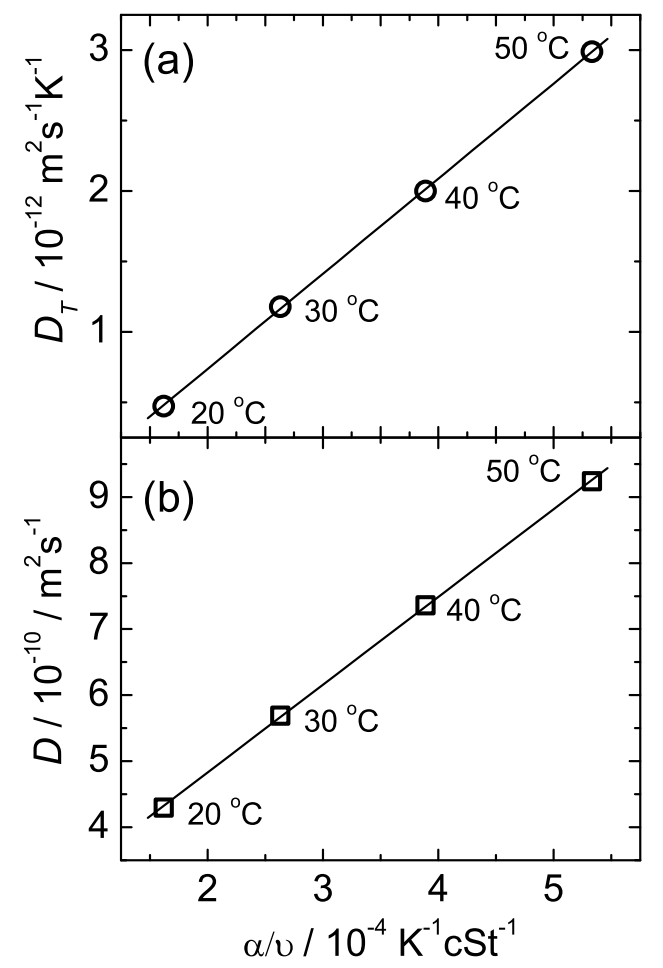

Figure 7: Influence of the temperature on the thermal diffusion (a) and mass diffusion (b) coefficients of D-glucose/water with a sugar weight fraction of $c=0.2$ as function of the ratio of the thermal expansion coefficient $\alpha$ and the kinematic viscosity $v$. 


\section{Ternary mixture Water-Glucose-Mannose}

We studied the ternary system D-glucose/D-mannose/water with a total sugar weight fraction of $c=2 / 3$ at $50{ }^{\circ} \mathrm{C}$. The same weight fraction has also been studied for the binary systems Dglucose/water, D-mannose/water and D-fructose/water. We have chosen the ternary system Dglucose/D-mannose/water because the refractive index increments $(\partial n / \partial T, \partial n / \partial c)$ and the mass diffusion coefficient are almost equal (see Table 1). We varied the mass ratio of the sugars in the ternary system from 0 and 1, which corresponds with the binary systems D-mannose/water and D-glucose/water, respectively. We prepared three ternary mixtures with sugar ratios (glucose/mannose) of c=1:3, 1:1 and 3:1. The data of $S_{\mathrm{T}}, D$ and $D_{T}$ are listed in Table 3. These data can be considered as an average value of both sugars, D-glucose and D-mannose.

In the literature, a number of conflicting approaches exists for the description of thermal diffusion coefficients in multicomponent mixtures. ${ }^{44-47}$ In general a ternary system can be described using three different thermal diffusion coefficients. For our system these are two coefficients, which describe the diffusion of the two sugars in water and one which describes the diffusion of one sugar in the other. In the measurement signal we detected only one concentration mode, so that we were not able to separate the signal into different contributions. Therefore, we determined one averaged thermal diffusion coefficient, which is a weighted sum of the different sugars and also their tautomers.

We found a linear dependence of the Soret coefficient, $S_{\mathrm{T}}$, as function of the mass ratio of glucose to the total sugar content (c.f. Figure 8).

$$
\begin{aligned}
& S_{\mathrm{T}}=(1.54 \pm 0.01) \times 10^{-3} \mathrm{~K}^{-1}+ \\
& (-0.7 \pm 0.01) \times 10^{-3} \mathrm{~K}^{-1} \cdot\left[\frac{m_{\text {glucose }}}{\left(m_{\text {glucose }}+m_{\text {mannose }}\right)}\right]
\end{aligned}
$$

In this paper we show that the Soret coefficient could also be used to determine the concentrations of two sugars with the same molecular weight. On one hand, it is possible to determine the mass 
ratio between sugar and water by measuring the mass diffusion coefficient (c.f. Figure 5), and on the other hand, the Soret coefficient gives the ratio between sugars (c.f. Figure 8). In principle, a method such as the thermal field flow separation could be used to separate different tautomers, but as in other methods the sugars in solution will readily change from one modification to another. ${ }^{48}$

Table 3: Thermal diffusion, mass diffusion and Soret coefficients of D-glucose/Dmannose/water mixtures at a water weight fraction of $c=0.3333$ and a total sugar weight fraction of $c=0.6667$. All measurements have been performed at $T=50{ }^{\circ} \mathbf{C}$.

\begin{tabular}{c|c|c|c}
\hline \hline $\begin{array}{c}\text { D-glucose mass } \\
\text { fraction from } \\
\text { total sugar }\end{array}$ & $\begin{array}{c}S_{\mathrm{T}} \\
/ 10^{-3} \mathrm{~K}^{-1}\end{array}$ & $\begin{array}{c}D \\
/ 10^{-10} \mathrm{~m}^{2} \mathrm{~s}^{-1}\end{array}$ & $\begin{array}{c}D_{T} \\
/ 10^{-13} \mathrm{~m}^{2} \mathrm{~s}^{-1} \mathrm{~K}^{-1}\end{array}$ \\
\hline 0.25 & $1.36 \pm 0.04$ & $2.52 \pm 0.08$ & $3.44 \pm 0.21$ \\
0.5 & $1.20 \pm 0.02$ & $2.45 \pm 0.09$ & $2.94 \pm 0.11$ \\
0.75 & $1.01 \pm 0.02$ & $2.51 \pm 0.06$ & $2.55 \pm 0.11$ \\
\hline \hline
\end{tabular}

\section{Conclusion}

In this work the thermal diffusion properties of aqueous solutions of monosaccharides are presented. The mass diffusion coefficient of the sugar is constant for a fixed concentration and temperature, and for the selected sugars independent of the sugar in the considered concentration and temperature range. Therefore, the molecular structure of the sugar does not affect the mass diffusion.

On the other hand the ketohexoses have higher thermal diffusion and Soret coefficients than the aldohexoses. It is complicated to find an explanation based on the molecular structure for the differences in the Soret and thermal diffusion coefficients. Only for some sugars we found a correlation between the thermal diffusion properties and the optical rotation. A clear correlation we find between the thermal diffusion coefficient and the ratio of the thermal expansion coefficient and the kinematic viscosity. Although we find only for the low concentration of $c=0.2$ a linear dependence we find at a higher concentration of $c=2 / 3$ a clear decay of $S_{\mathrm{T}}$ with $(\alpha / v)$ with small deviations from the linear behavior, which might be consequence of sugar-sugar interactions. 


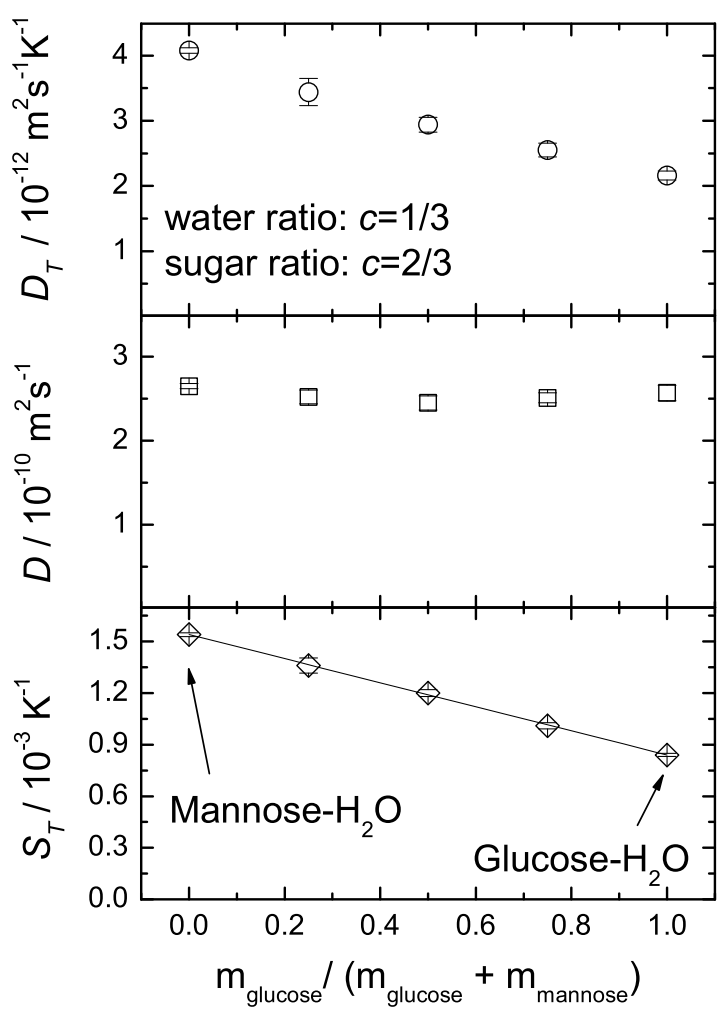

Figure 8: Soret coefficient of the system D-glucose/D-mannose/water as function of the D-glucose weight ratio to the total sugar content. The weight fraction of water is $c=1 / 3$, the total sugar weight fraction is $c=2 / 3$. All measurements have been performed at $T=50{ }^{\circ} \mathrm{C}$. The solid line corresponds to a linear fit of $S_{\mathrm{T}}$ as function of the sugar composition (c.f. Eq. 3). 
In the investigated range $D, D_{\mathrm{T}}$ and $S_{\mathrm{T}}$ increase with temperature and decrease with the sugar content. The relative differences in the thermal diffusion and Soret coefficient for different sugars become smaller for lower concentrations. The physical reason might be that the conformation of the molecule is more important if less space is available for each molecules and a denser packing is required.

From the studied ternary system, one can conclude that it could be possible to determine the sugar concentrations in aqueous solutions by thermal diffusion measurements. From the mass diffusion coefficient, one could determine the mass ratio between total sugar and water, and from the Soret coefficient one could determine the concentration of each sugar.

\section{Acknowledgement}

We appreciate many fruitful discussions with Pavel Polyakov, Malte Kleemeier and Gerd Meier. We thank Stefanie Jacob and Martina Bischop for their help with polarimeter measurements. We are also grateful for very helpful remarks by Stefan Immel. We thank the Department of Education, Universities and Investigation of Basque Government for the grant BFI08188.0. Financial support due to the Deutsche Forschungsgemeinschaft grant Wi 1684 is gratefully acknowledged.

\section{References}

(1) Vilar, J. M. G.; Rubi, J. M. Proc Natl Acad Sci USA 2001, 98, 11081-11084.

(2) Rubi, J. M. Sci. Am. 2008, 299, 62-67.

(3) Rubi, M. Meeting the entropy challenge 2008, 1033, 219-222.

(4) Rubi, J. M.; Naspreda, M.; Kjelstrup, S.; Bedeaux, D. J. Non-Equilib. Thermodyn. 2007, 32, $351-377$.

(5) Blanco, P.; Bou-Ali, M. M.; Platten, J. K.; Madariaga, J. A.; Urteaga, P.; Santamaria, C. J. Non-Equilib. Thermodyn. 2007, 32, 309-317. 
(6) Blanco, P.; Polyakov, P.; Bou-Ali, M. M.; Wiegand, S. J. Phys. Chem. B 2008, 112, 83408345.

(7) Debuschewitz, C.; Köhler, W. Phys. Rev. Lett. 2001, 87, 055901.

(8) Blanco, P.; Bou-Ali, M. M.; Platten, J. K.; Urteaga, P.; Madariaga, J. A.; Santamaria, C. J. Chem. Phys. 2008, 129, 174504.

(9) Polyakov, P.; Luettmer-Strathmann, J.; Wiegand, S. J. Phys. Chem. B 2006, 110, 2621526224.

(10) Wittko, G.; Köhler, W. EPL 2007, 78, 46007.

(11) Angeli, C.; Leonardi, E. Int. J. Heat Mass Transfer 2005, 48, 4633-4639.

(12) Bahat, A.; Tur-Kaspa, I.; Gakamsky, A.; Giojalas, L. C.; Breitbart, H.; Eisenbach, M. Nat. Med. 2003, 9, 149-150.

(13) Eisenbach, M.; Giojalas, L. C. Nat. Rev. Mol. Cell Biol. 2006, 7, 276-285.

(14) Braun, D.; Libchaber, A. Phys. Rev. Lett. 2002, 89, 188103.

(15) Montel, F.; Caillet, G.; Pucheu, A.; Caltaginore, J. P. Mar. Pet. Geol. 1993, 10, 51-57.

(16) Montel, F. Rev. Inst. Fr. Pet. 1998, 53, 9-11.

(17) Georis, P.; Legros, J. C.; Montel, F. Space Technology and Applications International Forum 1999, 458, 860-866.

(18) Sugaya, R.; Wolf, B. A.; Kita, R. Biomacromolecules 2006, 7, 435-440.

(19) Bourne, J. R.; Hegglin, M.; Prenosil, J. E. Biotechnol. Bioeng. 1983, 25, 1625-1639.

(20) Lindhorst, T. K. Essential of carbohydrate chemistry and biochemistry; WILEY-VCH, 2000.

(21) Wiegand, S.; Ning, H.; Kriegs, H. J. Phys. Chem. B 2007, 111, 14169-14174. 
(22) Thyagarajan, K.; Lallemand, P. Opt. Commun. 1978, 26, 54-57.

(23) Köhler, W. J. Chem. Phys. 1993, 98, 660-668.

(24) Köhler, W.; Rossmanith, P. J. Phys. Chem. 1995, 99, 5838-5847.

(25) Leppla, C.; Wiegand, S. Philos. Mag 2003, 83, 1989-1999.

(26) Köhler, W.; Schäfer, R. Polymer analysis by thermal-diffusion forced Rayleigh scattering. In New Developments in Polymer Analytics Ii; Schmidt, M., Ed.; Springer: Berlin, 2000; Vol. 151, pp 1-59.

(27) Wiegand, S.; Köhler, W. Measurement of Transport Coefficients by an optical grating technique. In Thermal Nonequilibrium Phenomena in Fluid Mixtures; Springer: Berlin, 2002; Vol. LNP584, pp 189-210.

(28) Sengers, J. V.; Watson, J. T. R. J. Phys. Chem. Ref. Data 1986, 15, 1291-1314.

(29) Spieweck, F.; Bettin, H. Tech. Mess. 1992, 59, 285-292.

(30) Becker, A.; Köhler, W.; Müller, B. Ber. Bunsen-Ges. Phys. Chem. Chem. Phys. 1995, 99, $600-608$.

(31) Schneider, B.; Lichtenthaler, F. W.; Steinle, G.; Schiweck, H. Liebigs Ann. Chem. 1985, $2443-2453$.

(32) Lichtenthaler, F. W.; Rönninger, S. J. Chem. Soc., Perkin Trans. 2 1990, 1489-1497.

(33) Polavarapu, P. L. Chirality 2002, 14, 768-781.

(34) da Silva, C. O.; Mennucci, B. J. Chem. Theory Comput. 2007, 3, 62-70.

(35) Hudson, C. S.; Yanovsky, E. J. Am. Chem. Soc. 1917, 39, 1013-1038.

(36) Pigman, W. W.; Isbell, H. S. J. Res. Nat. Bur. Stand. 1937, 19, 443-445. 
(37) Belousov, V. P.; Panov, M. Y. Thermodynamic properties of aqueous solutions of organic substances.; CRC Press: Boca Raton, FL, 1994.

(38) Iacopini, S.; Rusconi, R.; Piazza, R. Eur. Phys. J. E 2006, 19, 59-67.

(39) Brenner, H. Phys. Rev. E 2006, 74, year.

(40) Würger, A. Langmuir 2009, 25, 6696-6701.

(41) Hartung, M.; Rauch, J.; Köhler, W. J. Chem. Phys. 2006, 125, 214904.

(42) Shah, N. O.; Nickerson, T. A. Journal of food science 1978, 43, 1081-1084.

(43) Mogi, N.; Sugai, E.; Fuse, Y.; Funazukuri, T. J. Chem. Eng. Data 2007, 52, 40-43.

(44) Kempers, L. J. Chem. Phys. 1989, 90, 6541-6548.

(45) Vaerenbergh, S. V.; Legros, J. Entropie 1996, 198/199, 77.

(46) Larre, J. P.; Platten, J. K.; Chavepeyer, G. Int. J. Heat Fluid Fl. 1997, 40, 545-555.

(47) Firoozabadi, A.; Ghorayeb, K.; Shukla, K. Aiche J. 2000, 46, 892-900.

(48) Isbell, H. S.; Pigman, W. W. J. Res. Nat. Bur. Stand. 1938, 20, 773-798. 


\section{Graphical TOC Entry}

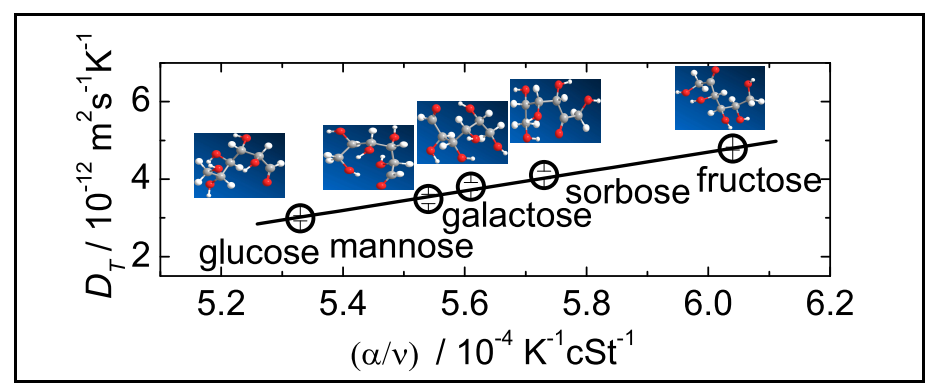

